\title{
« Reproductions et transplantations de lieux sacrés dans l'histoire du christianisme (Moyen Âge - époque moderne) »
}

\section{Ludovic Viallet}

\section{OpenEdition}

\section{Journals}

Édition électronique

URL : http://journals.openedition.org/ifha/126

DOI : 10.4000/ifha.126

ISSN : 2198-8943

\section{Éditeur}

IFRA - Institut franco-allemand (sciences historiques et sociales)

\section{Édition imprimée}

Date de publication : 6 février 2011

Pagination : 43-49

ISSN : 2190-0078

\section{Référence électronique}

Ludovic Viallet, « «Reproductions et transplantations de lieux sacrés dans I'histoire du christianisme (Moyen Âge - époque moderne) » », Revue de l'IFHA [En ligne], 3 | 2011, mis en ligne le 01 octobre 2012, consulté le 20 avril 2019. URL : http://journals.openedition.org/ifha/126 ; DOI : 10.4000/ifha.126

Ce document a été généré automatiquement le 20 avril 2019

(CIFHA 


\title{
"Reproductions et transplantations de lieux sacrés dans l'histoire du christianisme (Moyen Âge - époque moderne)"
}

\author{
Ludovic Viallet
}

\section{NOTE DE L'ÉDITEUR}

Colloque international, Clermont-Ferrand, 26-27 novembre 2010

1 Autour de la problématique des reproductions et transplantations occidentales de lieux sacrés, c'est toute une réflexion précise sur les rapports à l'espace et au sacré, et sur leurs usages, qu'a abordée ce colloque, organisé à Clermont-Ferrand par le Centre d'histoire «Espaces et cultures » de l'université Blaise-Pascal (ClermontFerrand II), en partenariat avec le Centre d'histoire sociale et culturelle de l'Occident (univ. Paris Ouest-Nanterre-La Défense) et l'Institut français d'histoire en Allemagne.

2 Les bornes chronologiques retenues ( $\mathrm{du}$ IXe au XVIIe siècle principalement) étaient vastes - bien que le propos ait fait la part belle au bas Moyen Âge -, de même que l'aire géographique considérée, établissant des connexions entre différents espaces occidentaux (France, Italie, Germanie, Europe centre-orientale) et les Lieux saints, ainsi qu'entre des échelles variées - de l'église jusqu'à la ville, voire aux réseaux européens. Cet ensemble étendu n'en possédait pas moins un arrière-plan commun : Jérusalem, et tout particulièrement le Saint-Sépulcre, démultiplié par ses images et reproductions, modèle par excellence de lieu transplanté. Les champs d'étude enfin étaient eux-mêmes divers, ayant trait tant à l'histoire des sensibilités et des pratiques religieuses qu'à celle des usages politiques et sociaux, ou encore à l'histoire de l'art - une diversité reflétée également par les sources convoquées, qui faisaient se répondre textes théoriques et de 
dévotion, récits de pèlerinages, cartes, objets, images et ensembles monumentaux et architecturaux. Malgré l'étendue de ces cadres et de ces approches, le choix qui a été fait par les organisateurs, Ludovic VIALLET et Laura GAFFURI, de restreindre le nombre des intervenants (tout en préservant leur caractère international) s'est révélé fructueux, permettant un resserrement des communications sur le cœur même du sujet du colloque.

Introduisant à la mécanique de « recréation » des Lieux saints, Ludovic VIALLET a rappelé qu'elle est déjà à l'œuvre dans certaines pratiques dévotionnelles, et pas uniquement dans les reproductions monumentales. Les unes comme les autres, à l'image des itinéraires du chemin de croix, peuvent impliquer une réelle " composition de lieu », selon l'expression reprise à Ignace de Loyola, qui réinsère dans l'espace des éléments autres. Les complexes reproduisant les Lieux saints n'ont donc pas seulement trait à l'espace qu'ils sacralisent, mais également au "voir» et au «croire ", participant ainsi d'un véritable «paysage culturel », ainsi qu'à la notion généralement peu goûtée par les historiens d'« imaginaire collectif »- entendue comme système assurant l'unité et la cohésion d'un groupe, non sans corrélation avec l'idéologie. Ils enracinent la piété dans l'espace et plus particulièrement, à la fin du Moyen Âge, dans l'espace urbain dans son ensemble, en mettant en scène l'identité poliade et en recréant un espace lointain, dès lors intégré à l'horizon familier des fidèles, eux-mêmes intégrés au drame sacré - non sans lien avec les pratiques théâtrales de ce temps des grands mystères religieux. De telles reproductions de lieux sacrés, qui créent autant d'espaces compensatoires, prennent le caractère d'hétérotopies par le jeu de renvois à d'autres espaces, mais aussi à l'idéal qui les soustend. Or, à l'âge d'une affirmation de l'emprise des autorités sur la ville, reléguant toute opposition à la marginalité, cet idéal implique une notion d'ordre et de contrôle : aussi ces transplantations et les dévotions qui leur sont liées sont-elles également à voir sous un angle moral et politique. En effet, cette mise en relation des lieux entre eux participe pour L. VIALLET de la construction de l'espace social, « dans laquelle un moteur essentiel a été l'imaginaire, nourri par la dévotion ».

Les trois communications suivantes se sont concentrées sur l'exemple du Saint-Sépulcre, à commencer par celle d'Élisabeth RUCHAUD concernant ses représentations et transferts durant la grande période de multiplication de ses imitations (IXe-XIe siècle). La centralité spirituelle du sépulcre christique, qu'elle a rappelée, explique la démultiplication de ses reproductions, moyen de sa diffusion en Occident. Variées dans leur forme, leur plan, leurs matériaux et leurs usages, ces représentations ne sont pas exactement fidèles à leur modèle : elles évoquent le saint tombeau d'abord par tout un ensemble de symboles et de références. Plutôt que des copies, ces imitations monumentales sous formes d'édicules, ou parfois d'églises entières, sont donc avant tout des reconstructions mentales, qui visent moins une reproduction à l'identique qu'une réappropriation symbolique du SaintSépulcre, traduit de multiples manières.

5 Peter KURManN a étudié l'un de ces exemples : la reproduction du Saint-Sépulcre dans la cathédrale de Constance. Enchâssée dans une rotonde comme le Sépulcre dans son anastasis, cette imitation a permis à Constance de se présenter au milieu du Xe siècle comme une nouvelle Jérusalem, au moment même où un programme architectural cherchait à en faire une seconde Rome, accumulant les renvois à d'autres lieux en un même site. Un nouvel édicule remplace cette première reproduction au milieu du XIIIe siècle et joue un rôle renouvelé dans la liturgie de la cathédrale, comme point d'aboutissement de fréquentes processions qui sont autant de transpositions locales $\mathrm{du}$ pèlerinage à Jérusalem. Le saint-sépulcre de Constance, également objet de méditation 
grâce aux images élaborées qui y sont sculptées, est ainsi le moyen d'un véritable "pèlerinage intérieur »; il donne à voir, sous forme architecturale, l'action centrale de l'histoire du salut, même loin des lieux qui l'ont vue s'accomplir.

6 Cette mise en relation directe avec les Lieux saints, Hans-Joachim SchmidT la retrouve dans les représentations du Saint-Sépulcre présentes dans les cartes et récits de pèlerinages médiévaux. En effet, si ceux-ci peuvent se faire très précis, évoquant presque toujours les mesures exactes du saint tombeau, c'est moins à titre purement descriptif que pour permettre une vénération des Lieux saints sans y être présent; la connaissance qu'ils en offrent et l'émotion qu'ils retranscrivent nourrissent alors l'imaginaire de Jérusalem et sa reconstruction mentale. Rendant présent l'absent, ils rendent là encore possible un pèlerinage intérieur, permis par cette ubiquité symbolique des lieux décrits, et sont un moyen de pallier l'antagonisme typiquement chrétien entre omniprésence du salut, qui suppose que chaque lieu soit équivalent dans l'obtention du salut, et concentration de la présence divine dans les Lieux saints.

7 Aux imitations du Saint-Sépulcre semblent succéder, aux XVe et XVIe siècles, la dévotion aux mises au tombeau monumentales du Christ, dont Elsa KARSALLAH a étudié les cas français. Ces groupes sculptés insistent sur la souffrance du Christ en se concentrant sur sa passion plutôt que sur sa résurrection, représentant son corps mort et meurtri plutôt que son absence du tombeau glorieux. Ils proposent donc de souffrir avec ceux qui ont assisté aux derniers instants du Christ, eux-mêmes présentés dans ces formes d'imagessynthèses qui condensent l'événement biblique. Reproduisant tant les lieux que l'action de la mise au tombeau, ces ensembles monumentaux ont même obtenu un statut comparable à leur modèle, puisque la visite à ces groupes faisait l'objet d'indulgences, accordant ainsi à l'image un statut presque équivalent à celui de relique et faisant $\mathrm{du}$ groupe sculpté un possible substitut du pèlerinage au Saint-Sépulcre lui-même.

De la transplantation du Saint-Sépulcre à celle de l'ensemble de la Ville sainte, les autres communications ont opéré un changement d'échelle d'analyse, s'attachant à étudier les reproductions et réappropriations de ces lieux sur de plus vastes ensembles. Les sacri monti en font partie, qui assurent une vision globale des Lieux saints et de l'histoire sainte, comme l'a montré Anne LEPOITTEVIN en s'intéressant plus particulièrement au sacro monte de Varallo. La reproduction revendique cette fois-ci une réelle similitude avec son modèle, notamment par ses dimensions, similitude qui confond la copie et l'original au point que le sacro monte est qualifié de «lieu saint ", de "nova Ierusalem » et fait luimême l'objet d'un pèlerinage. Là encore, ce ne sont pas les lieux seuls qui sont reproduits, mais également les personnages qui les ont occupés, à l'instar du Christ gisant présenté dans son sépulcre qui devrait être vide. L'intégration et la prolifération de statues peintes change dès lors la perception de ces reproductions monumentales, en insistant non seulement sur les lieux, mais également sur les événements, les épisodes de la vie du Christ ; la reproduction des lieux sacrés prime peut-être moins ici, avec le temps, que la présentation de l'histoire sainte.

9 Dans son analyse des grilles de chapelle pour ces mêmes sacri monti, Pierre-Antoine Fabre a insisté précisément sur ce rapport à l'histoire et surtout à la mémoire. Il voit dans ces recréations des Lieux saints un rôle de reconstruction du passé dans une mémoire immédiate du présent, mais aussi dans une forme de mémoire collective. Les grilles de chapelle impliquent alors que cette mémoire se constitue dans un rapport latéral à l'image et par une déambulation. Elles font des sacri monti des lieux de passage où il ne s'agit pas de rester, là où, à l'inverse, la vénération du Saint-Sépulcre supposait souvent 
d'y passer un certain temps. Malgré la revendication d'une similitude avec son modèle, la reproduction du lieu sacré implique donc une dévotion et un rapport différents, renouvelés par rapport à l'original.

10 Halina MANIKOWSKA proposait enfin d'étendre cette étude des transplantations de lieux sacrés à des ensembles encore plus vastes, en détaillant trois modalités différentes de transposition de Jérusalem au sein de villes entières dans l'Europe centre-orientale du Bas Moyen Âge : Prague, Görlitz et Breslau (Wrocław). Dans la première, c'est un programme d'aménagement d'ensemble, reprenant l'organisation hiérosolymitaine, l'axe de ses rues, ses mesures, ses grands monuments, qui a permis à la cité de se présenter comme nouvelle "ville sainte». À Görlitz, le même titre est revendiqué surtout via une reproduction ponctuelle des principaux Lieux saints, en recréant une chapelle idéalisée du Saint-Sépulcre, du Golgotha, ou encore un jardin des Oliviers. Enfin, à Breslau, cette transposition de Jérusalem s'est faite moins dans l'architecture que dans les pratiques dévotionnelles et pénitentielles, associées à l'octroi d'indulgences, dans une mise en scène de l'histoire biblique qui n'est pas sans rappeler les sacri monti italiens et leur statuaire, et surtout par l'instauration d'un chemin de croix et de processions qui réactualisaient, en la théâtralisant, la vie du Christ. Cet accent mis sur les rituels rappelle que l'assimilation à la Ville sainte n'est pas seulement statique, inscrite dans l'architecture monumentale, mais se crée également par tout un jeu de pratiques dévotionnelles, qui sont autant d'occasions de s'approprier l'espace urbain.

11 Laura GAFFURI et Paulo cozzo se sont enfin attachés à l'étude des usages politiques de ces transferts de sacralité au sein du duché de Savoie. La première a analysé la création des saintes-chapelles dans l'ensemble de l'Europe, avant de s'intéresser plus particulièrement à celle de Chambéry, créée au XVe siècle dans un duché alors en pleine affirmation. Incluse dans le château ducal, celle-ci illustre le lien étroit qui s'établit ici entre construction étatique et organisation de l'espace ecclésial, entre symbolique religieuse et idéologie politique. L. GAFFURI présente finalement la sainte-chapelle comme un modèle politique tout autant que religieux, une "icône sérielle » car reproduite et démultipliée, puisque la sainte-chapelle savoyarde s'inspire de celle de Saint Louis, notamment dans ses précieuses reliques, parmi lesquelles, pendant un temps, le saint suaire.

Ce dernier constitue précisément un pan entier du propos de P. cozzo dans sa communication sur la « reproduction de la sacralité mariale et sindonique » dans l'espace savoyard, cette fois à l'époque moderne. L'identité du duché s'y est construite selon lui autour du prestige de la dynastie ducale et de ses usages du sacré. Le rôle du saint suaire a été ici fondamental : relique majeure de la chrétienté et signe d'élection pour la famille des ducs, il a donc été l'objet de toute leur attention, visible dans l'appareil monumental et idéologique qui lui a été dédié, permettant notamment d'affirmer son authenticité face aux autres saints suaires alors revendiqués en Occident. Le suaire a également fait l'objet de copies authentifiées, elles-mêmes devenues de véritables reliques grâce au contact qu'elles ont entretenu avec l'original; ces reproductions, distribuées dans les grandes cours européennes, y assuraient le prestige des ducs de Savoie. P. cozzo a noté enfin l'importance du culte marial dans la formation identitaire du duché de Savoie à travers l'exemple du culte de Notre-Dame de Lorette, qui a nourri une intense dévotion locale au moment même où ce culte prenait un nouvel élan en Europe. Ainsi, de même qu'avec la reproduction du saint suaire la dynastie ducale cherchait à illustrer son élection en Occident, elle s'ancrait par l'usage du culte marial dans un réseau sacré à échelle européenne. 
Les conclusions proposées par Catherine VINCENT ont insisté sur l'importance des analyses communes qui se sont dessinées dans ce colloque. Les communications présentées ont souligné les différentes échelles de transplantations de lieux sacrés, de l'église à l'ensemble de l'espace urbain, qu'elles permettent de s'approprier. Leur usage est donc également social, et implique une grande variété d'acteurs parmi lesquels un rôle majeur est accordé aux Franciscains, mais aussi aux laïcs, à l'ensemble des fidèles qui animent cette transplantation. Les manifestations de cette dernière n'ont en effet rien de statique : elles sont l'objet de réaménagements et surtout de cérémonies, de processions, de déambulations. Non contentes d'être marquées dans les lieux, elles se traduisent également par tout un ensemble de pratiques dévotionnelles. D'autre part, le rôle central des lieux de la vie du Christ dans ces transplantations est une constante dans les communications présentées, de même que l'importance des mesures et des proportions que doivent respecter les reproductions. Celles-ci ne sont pour autant jamais ou presque de véritables copies, mais plutôt des réadaptations, composées d'ajouts, de modifications, signe d'une réappropriation des Lieux saints, dont la démultiplication permet de réactiver la mémoire du passé chrétien.

14 Cette conclusion met en avant les nombreuses idées communes auxquelles sont parvenus les intervenants, idées qui permettent de donner une vision d'ensemble de la question des transplantations et reproductions de lieux sacrés et d'en proposer déjà des éléments de synthèse. Aussi, loin de l'impression de cloisonnement et de fragmentation laissée par de trop nombreux colloques, on aura au contraire tiré profit ici de l'attention portée par chacun à la lettre du sujet. En ressort une réelle impression de réflexion d'ensemble et de dialogue entre des communications qui ont semblé se répondre, sans doute aidée par le resserrement du nombre de contributions et dont devrait rendre compte la publication à venir des actes de ce colloque. On aimerait saluer ce bel effort de cohésion, cette unité malgré la variété des études proposées - à l'image finalement de ces reproductions du Saint-Sépulcre, toutes différentes, mais qui se revendiquent d'un même modèle et d'un même esprit. 